\title{
Churg-Strauss Syndrome Presenting with Acute Renal Insufficiency Accompanied by Eosinophilic Tubulointerstitial Nephritis
}

\author{
Daigoro Hirohama ${ }^{1}$, Junichi Hoshino ${ }^{1}$, Keiichi Sumida ${ }^{1}$, Eiko Hasegawa ${ }^{1}$, \\ Rikako Hiramatsu ${ }^{1}$, Masayuki Yamanouchi ${ }^{1}$, Noriko Hayami ${ }^{1}$, Tatsuya Suwabe ${ }^{1}$, \\ Naoki Sawa ${ }^{1}$, Fumi Takemoto ${ }^{1}$, Yoshifumi Ubara ${ }^{1}$, Shigeko Hara ${ }^{2}$, \\ Kenichi Ohashi ${ }^{3}$ and Kenmei Takaichi ${ }^{1}$
}

\begin{abstract}
We encountered an unusual and rare case of 59-year-old woman with Churg-Strauss syndrome (CSS) showing myeloperoxidase anti-neutrophil cytoplasmic antibody (MPO-ANCA)-related acute renal insufficiency accompanied by eosinophilic tubulointerstitial nephritis. To date, reports in English of CSS presenting with rapidly progressive/acute renal insufficiency and biopsy-proven renal lesions have been uncommon. Here, we discuss this unusual case and review the previously reported CSS cases. The complication of eosinophilic tubulointerstitial nephritis in CSS cases with acute renal insufficiency might be higher than generally thought. Furthermore, the presence of eosinophilic infiltration and eosinophilic tubulointerstitial nephritis might be associated with the good renal outcome in CSS patients.
\end{abstract}

Key words: Churg-Strauss syndrome, acute renal insufficiency, eosinophilic tubulointerstitial nephritis, ANCA-associated vasculitis, MPO-ANCA

(Intern Med 51: 1555-1560, 2012)

(DOI: 10.2169/internalmedicine.51.6063)

\section{Introduction}

Churg-Strauss syndrome (CSS) is defined as an eosinophil-rich, granulomatous inflammation involving the respiratory tract and necrotizing vasculitis affecting small to medium-sized vessels, associated with asthma and eosinophilia $(1,2)$. In Europe, the annual prevalence and incidence rate of CSS are 6.8 and 2.4 per million $(3,4)$, whereas the numbers of patients and new cases are lower (1,800 and 100 per year, respectively) in Japan (5). Pathological confirmation is based on the presence of extra-vascular granulomas in association with necrosis and predominant extra-vascular eosinophils, as well as necrotizing vasculitis $(1,2)$. All of these lesions however, are rarely found together in biopsy specimens (6-9). To date, CSS presenting with rapidly pro- gressive/acute renal insufficiency and biopsy-proven renal lesions which were fully described in English-language literature have been rare. Moreover, these included some cases with eosinophilic tubulointerstitial nephritis. The gender distribution of CSS with rapidly progressive/acute renal insufficiency and eosinophilic tubulointerstitial nephritis is still unclear. Here, we report a rare case of a female CSS patient showing myeloperoxidase anti-neutrophil cytoplasmic antibody (MPO-ANCA)-related acute renal insufficiency accompanied by eosinophilic tubulointerstitial nephritis.

\section{Case Report}

A 59-year-old woman with a 2-year history of bronchial asthma (BA) presented with fever and fatigue for one month. In the past year, she had been exposed to no drugs

${ }^{1}$ Nephrology Center, Toranomon Hospital, Japan, ${ }^{2}$ Health Management Center, Toranomon Hospital, Japan and ${ }^{3}$ Department of Pathology, Toranomon Hospital, Japan

Received for publication June 25, 2011; Accepted for publication February 27, 2012

Correspondence to Dr. Daigoro Hirohama, hirohama-tky@umin.ac.jp 

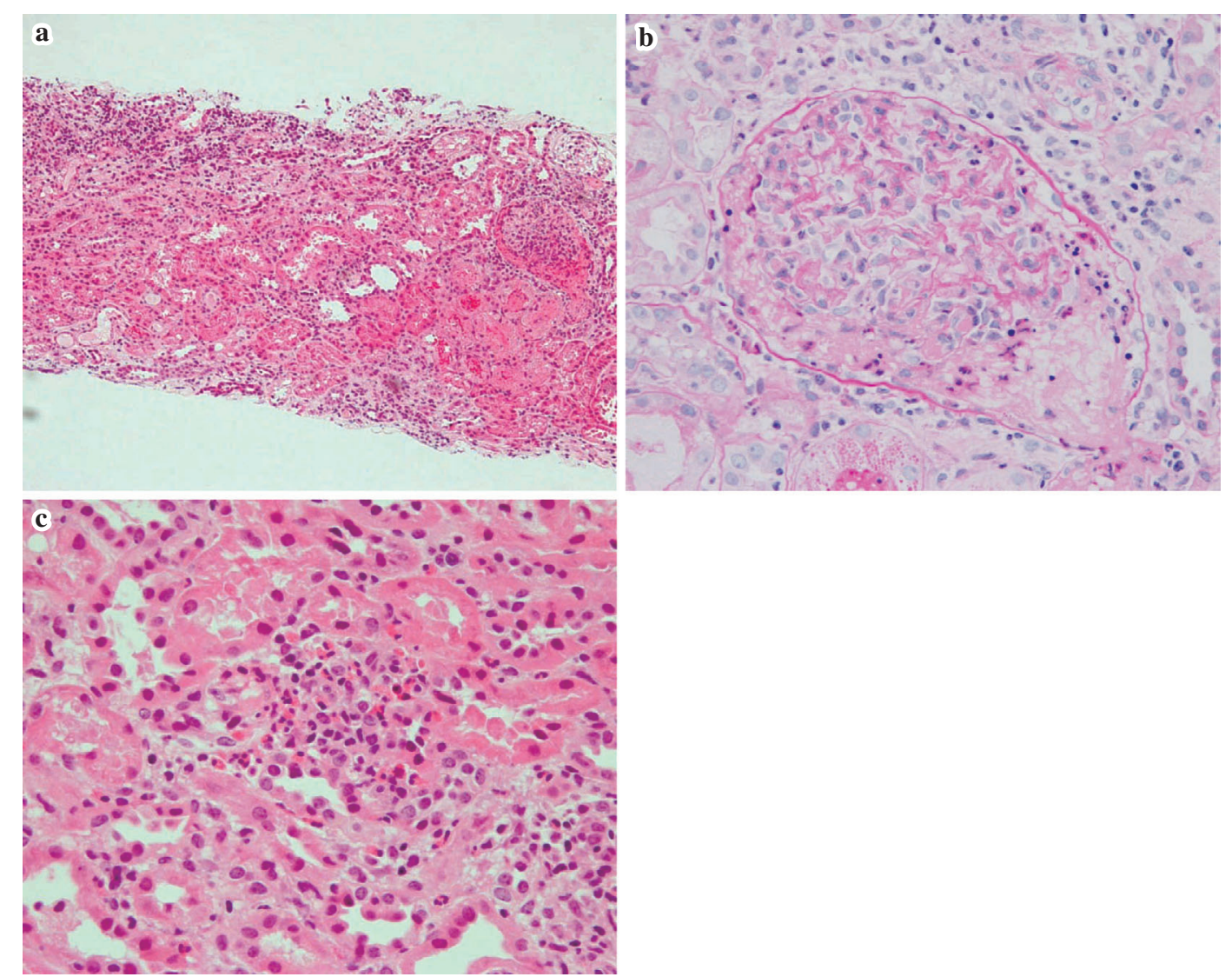

Figure 1. Light microscopic examination of the renal biopsy specimen showed a segmental necrotizing lesion with fibrin-rich material in the Bowman's space and tubulointerstitial nephritis with marked eosinophilic infiltration (a: Hematoxylin and Eosin staining; original magnification $\times 100$, b: periodic acid-Schiff staining; original magnification $\times 400$, c: Hematoxylin and Eosin staining; original magnification $\times \mathbf{4 0 0}$ ).

except for an inhaled corticosteroid (CS) for BA. A year before her admission, serum creatinine was $0.7 \mathrm{mg} / \mathrm{dL}$, and eosinophilia was not found. A week before her admission, serum creatinine was $0.9 \mathrm{mg} / \mathrm{dL}$.

On admission, her blood pressure and body temperature were $127 / 76 \mathrm{mmHg}$ and $38.1^{\circ} \mathrm{C}$, respectively. Cardiac and neurologic examinations showed normal findings. She had no extremity pitting edema, but reported the recent onset of purpura at both legs. Laboratory findings on admission were: white blood cell count, $14.5 \times 10^{3} / \mu \mathrm{L}$; eosinophils, $17 \%$ $(2,465$ cells $/ \mu \mathrm{L})$; hemoglobin, $10.5 \mathrm{~g} / \mathrm{dL}$; platelets, $343 \times 10^{3} /$ $\mu \mathrm{L}$; total protein, $6.7 \mathrm{~g} / \mathrm{dL}$; serum albumin, $2.4 \mathrm{~g} / \mathrm{dL}$; serum urea nitrogen, $21 \mathrm{mg} / \mathrm{dL}$; serum creatinine, $1.4 \mathrm{mg} / \mathrm{dL}$; serum uric acid, $3.6 \mathrm{mg} / \mathrm{dL}$; sodium, $136 \mathrm{mEq} / \mathrm{L}$; potassium, $4.2 \mathrm{mEq} / \mathrm{L}$; chloride, $100 \mathrm{mEq} / \mathrm{L}$; calcium, $8.0 \mathrm{mg} / \mathrm{dL}$; phosphate, $3.5 \mathrm{mg} / \mathrm{dL} ; \mathrm{IgG}, 1,612 \mathrm{mg} / \mathrm{dL} ; \mathrm{IgA}, 159 \mathrm{mg} / \mathrm{dL} ; \mathrm{IgM}$, $89 \mathrm{mg} / \mathrm{dL}$; IgE, $296 \mathrm{IU} / \mathrm{L}$; erythrocyte sedimentation rate, $>110 \mathrm{~mm} / \mathrm{h}$; and C-reactive protein, $18.6 \mathrm{mg} / \mathrm{dL}$. On serologic examination, her MPO-ANCA was elevated at 1,270 EU (normal <20 EU), proteinase 3-ANCA (PR3-ANCA) was negative at less than $10 \mathrm{EU}$. Anti-DNA antibody, antinuclear antibody, and anti-glomerular basement membrane antibody were not detected. Complement values were normal. Urinalysis showed many red blood cells and red blood cell casts. Urinary protein excretion was $1.1 \mathrm{~g} /$ day. Creatinine clearance was $25.6 \mathrm{~mL} / \mathrm{min}$.

Magnetic resonance imaging brain scans showed mucosal thickening of nasal sinuses, which was compatible with bilateral sinusitis. A computed tomographic chest scan showed no evidence of pulmonary vasculitis. A skin biopsy specimen taken from purpura of her right lateral lower thigh revealed perivascular dermatitis with eosinophilic infiltration. Renal ultrasound identified 2 normal-sized kidneys. CSS was considered, and renal biopsy was performed in order to establish a pathological diagnosis.

The renal biopsy specimen showed 19 glomeruli. In 2 of 19 glomeruli, segmental necrotizing lesions with fibrin-rich material in the Bowman's space were observed, and there were intraluminal eosinophils and neutrophils in the glomeruli (Fig. 1-a, b). There were slight degrees of fibrosis and marked infiltration of eosinophils in the interstitium (Fig. 1-c), but no granulomas were observed. Arteries and arterioles were slightly hyalinized but fibrinoid necrosis was not found. Immunofluorescence showed no immune deposits. We diagnosed it as an eosinophilic tubulointerstitial nephritis, rather than a pauci-immune type crescentic and necrotizing glomerulonephritis with severe eosinophilic interstitial infiltration, because the percentage of crescentic for- 


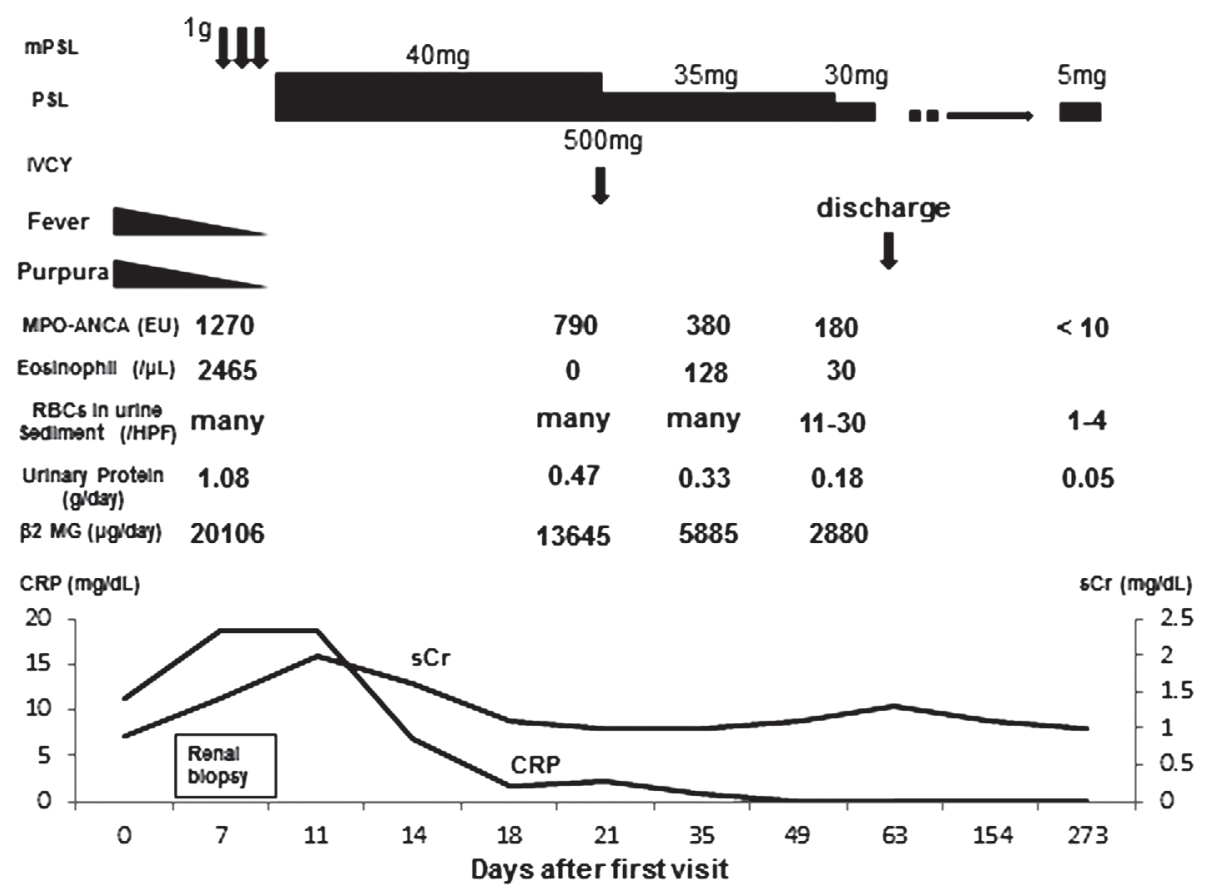

Figure 2. Clinical course after first visit. After treatment with pulses of intravenous methylprednisolone, oral prednisolone, and a high dose of intravenous cyclophosphamide, her clinical condition improved markedly. ANCA: antineutrophil cytoplasmic antibodies, 32 MG: Beta-2 microgloblin, CRP: C-reactive protein, IVCY: intraveneous cyclophosphamide, mPSL: methylprednisolone, PSL: prednisolone, $\mathrm{sCr}$ : serum creatinine

mation in Bowman's capsule was far less than $50 \%$, the number of affected glomeruli was less than $10 \%$, and the degree of eosinophilic infiltration was much more severe in the interstitium than in glomeruli. CSS was comprehensively diagnosed based on the presence of asthma, eosinophilia, sinusitis, and histological findings showing infiltration of many eosinophils to glomeruli and tubulointerstitium.

Treatment included pulses of intravenous methylprednisolone, oral prednisolone, and a high dose of intravenous cyclophosphamide. After immunosuppressive therapy, her clinical condition improved markedly. At outpatient review after 9 months in August 2010, her serum creatinine had decreased to $1.0 \mathrm{mg} / \mathrm{dL}$ without any hematuria, proteinuria, nor MPO-ANCA titers. She is now on a daily maintenance dose of $5 \mathrm{mg}$ prednisolone, and remains in good condition (Fig. 2).

\section{Discussion}

CSS is known as an allergic granulomatosis and angiitis, characterized by classic histopathological findings of vasculitis, and necrotizing extra-vascular granulomas with eosinophilic infiltration (1). However, these three histopathological findings rarely coexist, particularly in tissue biopsy specimens. Microscopic polyangitis, Wegener's granulomatosis and CSS also share pathologically identical pauci-immune systemic vasculitis (10). Moreover, since approximately $75 \%$ of patients with pauci-immune crescentic glomerulonephritis have overt evidence for systemic vasculitis, patients with pauci-immune crescentic glomerulonephritis with and without systemic vasculitis are indistinguishable (10).

Renal disease is often an overlooked feature of CSS. Although less frequent and less severe than in other forms of ANCA-associated vasculitis, nonetheless renal manifestations occur in $25-45 \%$ of CSS patients $(11,12)$. The most typical picture is pauci-immune focal and segmental necrotizing glomerulonephritis, with or without crescents, which usually involve $<50 \%$ of the glomeruli (11). In addition, since eosinophilic tubulointerstitial nephritis is not specific to CSS, clinicians always need to carefully carry out a comprehensive diagnosis.

Although renal manifestations are considered not rare in CSS patients, CSS presenting with rapidly progressive/acute renal insufficiency and biopsy-proven renal lesions which were fully described in English have been rare. To the best of our knowledge, these included 18 men and 12 women with ages ranging from 23 to 86 years (Table 1). Compared to the CSS incidence or prevalence rate mentioned above, remarkably few CSS cases have presented with rapidly progressive/acute renal insufficiency. A necrotizing crescentic glomerulonephritis was present in 25 patients (83\%). In described cases, extracapillary proliferation in more than $50 \%$ of glomeruli was present in 10 of 19 patients (53\%). An eosinophil-rich interstitial infiltration was present in 18 of 31 patients $(58 \%), 13$ of them had eosinophilic tubulointerstitial nephritis (patients 5, 6, 7, 8, 15, 17, 20, 21, 22, 23, 24,28 and the present patient), including 2 eosinophilic tubulointerstitial nephritis cases without glomerular involve- 
Table 1. Churg-Strauss Syndrome Presenting with Rapidly Progressive/Acute Renal Insufficiency and Biopsy-proven Renal Lesions

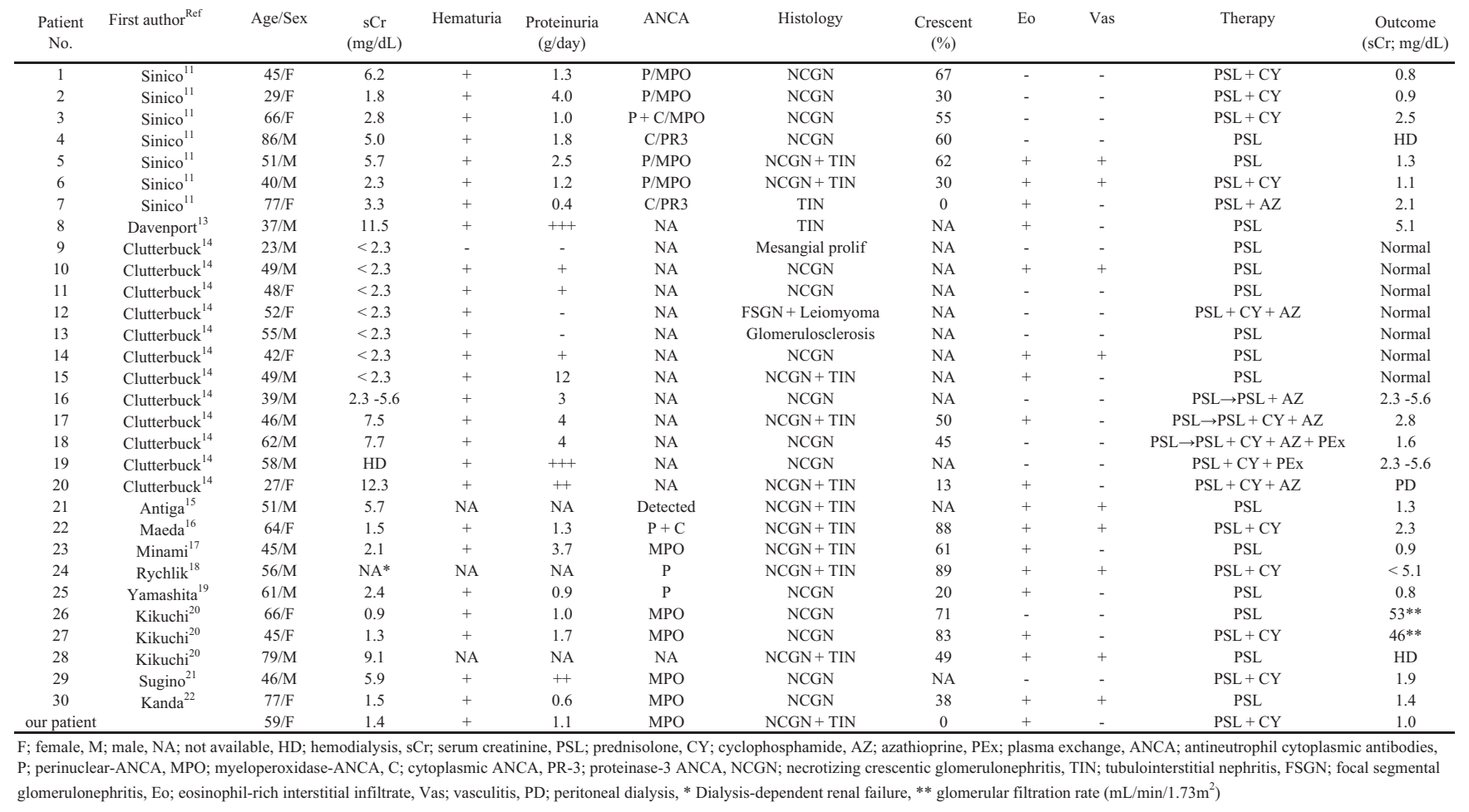

Table 2. The Renal Outcome of CSS Cases with or without Eosinophilic Tubulointerstitial Nephritis

\begin{tabular}{|c|c|c|c|c|}
\hline & & \multicolumn{2}{|c|}{$\begin{array}{l}\text { Recovery of renal } \\
\text { function }\end{array}$} & \multirow[t]{2}{*}{ Total } \\
\hline & & Yes & No & \\
\hline \multirow{2}{*}{$\begin{array}{l}\text { Eosinophilic } \\
\text { tubulointerstitial } \\
\text { nephritis }\end{array}$} & Yes & 10 & 3 & 13 \\
\hline & No & 14 & 1 & 15 \\
\hline \multirow[t]{2}{*}{ Total } & & 24 & 4 & 28 \\
\hline & & \multicolumn{3}{|c|}{ Fisher's exact test, $p=0.31$} \\
\hline
\end{tabular}

ment (patients 7 and 8). Necrotizing vasculitis was present in 9 of 31 patients $(29 \%$ ) (patients $5,6,10,14,21,22,24$, 28 and 30). All patients were treated with corticosteroids, in association with immunosuppressive drugs. All renal biopsies were performed at the time of diagnosis of CSS.

Guillevin et al. (7) reported 4 renal biopsy-performed CSS cases with segmental proliferative glomerulonephritis. Of these, 3 had crescentic lesions and 2 had renal insufficiency. However, none of them had vascular inflammation or extra-vascular eosinophil granulomas.

Concerning the renal outcome, 28 cases in which association between pre- and post-renal function was clearly referred, were available (excluding patients 16, 26 and 27). In this paper, recovery of renal function is defined as a decrease of serum creatinine. The renal outcome of CSS cases with rapidly progressive/acute renal insufficiency is generally good because only 3 of 28 patients (11\%) progressed to end-stage renal disease (patients 4, 20 and 28), and only 1 of 28 patients (4\%) showed no recovery of renal function (patient 22). On the other hand, 24 of 28 (86\%) CSS cases showed partial or complete recovery of renal function even if they had necrotizing crescentic glomerulonephritis (Table 1). Moreover, though it is rare (11), the renal outcome of CSS cases with eosinophilic tubulointerstitial nephritis might be worse than in cases without eosinophilic tubulointerstitial nephritis. In cases with or without eosinophilic tubulointerstitial nephritis, 10 of $13(77 \%)$ or 14 of 15 (93\%) cases showed partial or complete recovery of renal function, respectively (Fisher's exact test, $\mathrm{p}=0.31$ ) (Table 2). The complication of eosinophilic tubulointerstitial nephritis in CSS cases with acute renal insufficiency might be higher than generally thought. The presence of eosinophilic infiltration and eosinophilic tubulointerstitial nephritis might be associated with the good renal outcome. Eosinophils may cause tissue damage by releasing cytotoxic granule proteins (for example, eosinophil cationic and major basic protein) or by inducing apoptosis of target cells (23). Previously reported CSS cases with eosinophilic tubulointerstitial nephritis in which renal function did not recover were in end-stage renal disease at the time of diagnosis. Meanwhile in CSS cases as in the present case, in which eosinophilic tubulointerstitial nephritis was found at an early stage of renal insufficiency, the renal outcome might be better. The difference between eosinophilic tubulointerstitial nephritis with or without glomerular involvement in CSS remains unclear. Although most of the renal involvement of CSS is mild and not rare $(45 \%)(10)$, the number of reported CSS patients with acute renal insufficiency is small. Hence, it is reason- 
able to assume that CSS patients with glomerular involvement tend to have severe interstitial nephritis, and, as a result, have a worse renal outcome.

In any event, the clinical condition in CSS cases with rapidly progressive or acute renal insufficiency can most likely be improved by immunosuppressive therapy. Thus, it is important for detection and treatment to be carried out at an early stage.

In a previous study of 82 CSS patients (and 260 polyarteritis nodosa) (24), the French Vasculitis Study Group (FVSG) identified 5 factors that were significantly associated with a poor outcome (Five-Factors Score, FFS): elevated serum creatinine levels $(>140 \mu \mathrm{mol} / \mathrm{L}$, or $1.58 \mathrm{mg} / \mathrm{dL})$, proteinuria ( $>1 \mathrm{~g} /$ day), severe gastrointestinal tract involvement, cardiomyopathy, and/or central nervous system involvement. According to that analysis, when one factor was present at disease onset, as in the present case, the 5-year mortality rate was $25.9 \%$. Current views indicate that the treatment of CSS should be tailored on the basis of patient prognosis. Patients with $\mathrm{FFS}=0$ should receive $\mathrm{CS}$ alone, whereas those with FFS $>0$ should also receive cytotoxic agents (for example, cyclophosphamide, CY) as first-line therapy $(4,25-27)$. High CS doses (usually $1 \mathrm{mg} / \mathrm{kg} /$ day of prednisone, PSL) are used as initial therapy, with CS pulses before oral treatment in severe cases. CY is also added to $\mathrm{CS}$ in cases of steroid-resistant, steroid-dependent, or frequently relapsing diseases $(25,26)$. As for the duration of induction therapy, it has been shown that patients given $\mathrm{CY}$ for 4-6 months have higher relapse rates than those treated for 1 year (26). According to the European Vasculitis Study Group, following the induction of remission, intermittent high-dose intravenous CY achieves similar or better outcomes than oral CY, and is associated with lower recurrence rates (28). In the present case, the decision was made to administer CS pulses, followed by oral PSL at $40 \mathrm{mg}(0.8 \mathrm{mg} /$ $\mathrm{kg}$ )/day. Furthermore, intermittent high-dose intravenous $\mathrm{CY}$ was administered in addition to CS pulse therapy, as her FFS was 1.

In this report, we could not perform immunostaining using the renal biopsy specimen. This was a limitation, however, our pathological findings suggest the proportion of infiltrating cells other than eosinophils is rare. In conclusion, the complication of eosinophilic tubulointerstitial nephritis in CSS cases with acute renal insufficiency might be higher than generally thought. Furthermore, the presence of eosinophilic infiltration and eosinophilic tubulointerstitial nephritis might be associated with the good renal outcome in CSS patients. Although CSS often responds to CS and immunosuppressants, more selective therapeutic approaches are needed to reduce exposure to immunosuppression and for refractory cases. Future studies are warranted to elucidate the genetic and immune-mediated pathogenetic mechanisms.

The authors state that they have no Conflict of Interest (COI).

\section{Acknowledgement}

This study was funded by the Okinaka Memorial Institute for Medical Research.

\section{References}

1. Churg J, Strauss L. Allergic granulomatosis angiitis and periarteritis nodosa. Am J Pathol 27: 277-294, 1951.

2. Jennette JC, Falk RJ, Andrassy K, et al. Nomenclature of systemic vasculitides: Proposal of an international consensus conference. Arthritis Rheum 37: 187-192, 1994.

3. Martin RM, Wilton LV, Mann RD. Pharmacoepidemiol Drug Saf 8: 179-189, 1999.

4. Bosch X, Guilabert A, Espinosa G, Mirapeix E. Treatment of antineutrophil cytoplasmic antibody associated vasculitis: a systematic review. JAMA 298: 655-669, 2007.

5. Japan Intractable Diseases Information Center [internet], 2011. Available from http://www.nanbyou.or.jp/entry/208. Accessed 11 February 2012, (in Japanese).

6. Lanham JG, Elkon KB, Pusey CD, Hughes GR. Systemic vasculitis with asthma and eosinophilia: A clinical approach to the Churg-Strauss syndrome. Medicine (Baltimore) 63: 65-81, 1984.

7. Guillevin L, Cohen P, Gayraud M, Jarrousse B, Casassus P. Churg-Strauss syndrome: Clinical study and long term follow-up of 96 patients. Medicine (Baltimore) 78: 26-37, 1999.

8. Eustace JA, Nadasdy T, Choi M. The Churg Strauss syndrome. J Am Soc Nephrol 10: 2048-2055, 1999.

9. Noth I, Strek ME, Leff AR. Churg-Strauss syndrome. Lancet 361: 587-594, 2003.

10. Janette JC, Olsor JL, Schwartz MM, Silva FG. Heptinstall's Pathology of the Kidney. 6th ed. Lippincott Williams \& Wilkins, Philadelphia, 2007: 644-645.

11. Sinico RA, Di Toma L, Maggiore U, et al. Renal involvement in Churg-Strauss syndrome. Am J Kidney Dis 47: 770-779, 2006.

12. Jennette JC, Falk RJ. Small-vessel vasculitis. N Engl J Med 337: 1512-1523, 1997.

13. Davenport A, McDicken I, Goldsmith HJ. Reversible acute renal failure due to Churg-Strauss syndrome. Postgrad Med J 64: 713 $715,1988$.

14. Clutterbuck EJ, Evans DJ, Pusey CD. Renal involvement in Churg-Strauss syndrome. Nephrol Dial Transplant 5: 161-167, 1990.

15. Antiga G, Volpi A, Battini G, et al. Acute renal failure in ChurgStrauss syndrome: A case report. Contrib Nephrol 94: 177-180, 1991.

16. Maeda $Y$, Tomura $S$, Kato $K$, et al. Churg-Strauss syndrome associated with necrotizing crescentic glomerulonephritis in a diabetic patient. Intern Med 36: 68-72, 1997.

17. Minami J, Ishibashi-Ueda H, Okano Y, et al. Crescentic glomerulonephritis and elevated anti-myeloperoxidase antibody in a patient with Churg-Strauss syndrome. Nephron 77: 105-108, 1997.

18. Rychlik I, Tesar V, Stejskalova A, Stejskal J, Honsova E, Bartunkova J. ANCA-positive Churg-Strauss syndrome with renal failure. Nephrol Dial Transplant 12: 837-838, 1997.

19. Yamashita Y, Yorioka N, Taniguchi Y, et al. Nonasthmatic case of Churg-Strauss syndrome with rapidly progressive glomerulonephritis. Intern Med 37: 561-563, 1998.

20. Kikuchi Y, Ikehata N, Tajima O, Yoshizawa N, Miura S. Glomerular lesions in patients with Churg-Strauss syndrome and the antimyeloperoxidase antibody. Clin Nephrol 55: 429-435, 2001.

21. Sugino K, Kikuchi N, Muramatsu Y, et al. Churg-Strauss syndrome presenting with diffuse alveolar hemorrhage and rapidly progressive glomerulonephritis. Intern Med 48: 1807-1811, 2009.

22. Kanda T, Tanio H, Wu C, Nishihara H, Nogaki F, Ono T. ChurgStrauss syndrome with severe granulomatous angiitis and crescentic glomerulonephritis, which developed during therapy with a leu- 
kotriene receptor antagonist. Clin Exp Nephrol 14: 602-607, 2010.

23. Corradi D, Vaglio A, Maestri R, et al. Eosinophilic myocarditis in a patient with idiopathic hypereosinophilic syndrome: insights into mechanisms of myocardial cell death. Hum Pathol 35: 1160-1163, 2004.

24. Guillevin L, Lhote F, Gayraud M, et al. Prognostic factors in polyarteritis nodosa and Churg-Strauss syndrome: a prospective study in 342 patients. Medicine (Baltimore) 75: 17-28, 1996.

25. Pagnoux C, Guilpain P, Guillevin L. Churg-Strauss syndrome. Curr Opin Rheumatol 19: 25-32, 2007.

26. Cohen P, Pagnoux C, Mahr A, et al; French Vasculitis Study Group. Churg-Strauss syndrome with poorprognosis factors: a pro- spective multicenter trial comparing glucocorticoids and six or twelve cyclophosphamide pulses in forty-eight patients. Arthritis Rheum 57: 686-693, 2007.

27. Ribi C, Cohen P, Pagnoux C, et al; French Vasculitis Study Group. Treatment of Churg-Strauss syndrome without poorprognosis factors: a multicenter, prospective, randomized, openlabel study of seventy-two patients. Arthritis Rheum 58: 586-594, 2008.

28. de Groot K, Adu D, Savage COS; EUVAS (European vasculitis study group). The value of pulse cyclophosphamide in ANCA associated vasculitis: meta-analysis and critical review. Nephrol Dial Transplant 16: 2018-2027, 2001.

(C) 2012 The Japanese Society of Internal Medicine http://www.naika.or.jp/imindex.html 